\title{
Radiation-Induced Chemical Dynamics in Ar Clusters Exposed to Strong X-Ray Pulses
}

\author{
Yoshiaki Kumagai, ${ }^{1}$ Zoltan Jurek, ${ }^{2,3}$ Weiqing Xu,${ }^{1,4}$ Hironobu Fukuzawa, ${ }^{1,5}$ Koji Motomura, ${ }^{1}$ Denys Iablonskyi, \\ Kiyonobu Nagaya, ${ }^{5,6}$ Shin-ichi Wada, ${ }^{5,7}$ Subhendu Mondal, ${ }^{1}$ Tetsuya Tachibana, ${ }^{1}$ Yuta Ito, ${ }^{1}$ Tsukasa Sakai, ${ }^{6}$ \\ Kenji Matsunami ${ }^{6}$ Toshiyuki Nishiyama, ${ }^{6}$ Takayuki Umemoto, ${ }^{7}$ Christophe Nicolas, ${ }^{8}$ Catalin Miron, ${ }^{8,9,10}$ \\ Tadashi Togashi, ${ }^{11}$ Kanade Ogawa, ${ }^{5}$ Shigeki Owada, ${ }^{5}$ Kensuke Tono, ${ }^{11}$ Makina Yabashi, ${ }^{5}$ Sang-Kil Son, ${ }^{2,3}$ \\ Beata Ziaja, ${ }^{2,3}, 12$ Robin Santra, ${ }^{2,3,13}$ and Kiyoshi Ueda ${ }^{1,5}$ \\ ${ }^{1}$ Institute of Multidisciplinary Research for Advanced Materials, Tohoku University, Sendai 980-8577, Japan \\ ${ }^{2}$ Center for Free-Electron Laser Science, Deutsches Elektronen-Synchrotron, Notkestrasse 85, 22607 Hamburg, Germany \\ ${ }^{3}$ The Hamburg Centre for Ultrafast Imaging, Luruper Chaussee 149, 22671 Hamburg, Germany \\ ${ }^{4}$ School of Physical Science and Technology, ShanghaiTech University, Shanghai 201210, China \\ ${ }^{5}$ RIKEN SPring-8 Center, Sayo, Hyogo 679-5148, Japan \\ ${ }^{6}$ Department of Physics, Kyoto University, Kyoto 606-8502, Japan \\ ${ }^{7}$ Department of Physical Science, Hiroshima University, Higashi-Hiroshima 739-8526, Japan \\ ${ }^{8}$ Synchrotron SOLEIL, L'Orme des Merisiers, Saint-Aubin, BP 48, FR-91192 Gif-sur-Yvette Cedex, France \\ ${ }^{9}$ Extreme Light Infrastructure-Nuclear Physiscs (ELI-NP), "Horia Hulubei" National Institute for Physics and Nuclear Engineering, \\ 30 Reactorului Street, RO-077125 Măgurele, Jud. Ilfov, Romania \\ ${ }^{10}$ LIDYL, CEA, CNRS, Université Paris-Saclay, CEA Saclay, 91191 Gif-sur-Yvette, France \\ ${ }^{11}$ Japan Synchrotron Radiation Research Institute (JASRI), Sayo, Hyogo 679-5198, Japan \\ ${ }^{12}$ Institute of Nuclear Physics, PAS, Radzikowskiego 152, 31-342, Krakow, Poland \\ ${ }^{13}$ Department of Physics, University of Hamburg, Jungiusstrasse 9, 20355 Hamburg, Germany
}

(Received 30 September 2017; revised manuscript received 30 January 2018; published 31 May 2018)

\begin{abstract}
We show that electron and ion spectroscopy reveals the details of the oligomer formation in Ar clusters exposed to an x-ray free electron laser (XFEL) pulse, i.e., chemical dynamics triggered by $\mathrm{x}$ rays. With guidance from a dedicated molecular dynamics simulation tool, we find that van der Waals bonding, the oligomer formation mechanism, and charge transfer among the cluster constituents significantly affect ionization dynamics induced by an XFEL pulse of moderate fluence. Our results clearly demonstrate that XFEL pulses can be used not only to "damage and destroy" molecular assemblies but also to modify and transform their molecular structure. The accuracy of the predictions obtained makes it possible to apply the cluster spectroscopy, in connection with the respective simulations, for estimation of the XFEL pulse fluence in the fluence regime below single-atom multiple-photon absorption, which is hardly accessible with other diagnostic tools.
\end{abstract}

DOI: 10.1103/PhysRevLett.120.223201

Ultrashort pulses from x-ray free electron lasers (XFELs) $[1,2]$ enable unique studies of structural dynamics, permitting us to determine so far unknown structures of, e.g., protein molecules [3,4] and transient species [5] and to probe light-induced structural transitions [6-8]. In parallel, investigations of the interaction between matter and XFEL pulses in a new regime of $\mathrm{x}$-ray intensity open novel avenues in atomic, molecular, and solid state physics [8-13]. In general, the ultrafast reactions induced by an XFEL pulse are of fundamental interest as well as of crucial importance when employing XFEL for structure determination $[14,15]$. In spite of this strong need, XFEL-induced chemical transformation of large molecular assemblies has not been investigated in much detail so far.

Here, we report on a recent experiment performed at SACLA, an XFEL in Japan, which reveals the details of the oligomer formation, following the irradiation of $\mathrm{Ar}_{1000}$ clusters. Clusters composed of Ar atoms were irradiated by femtosecond hard-x-ray pulses. The kinetic energy spectra of electron and ionic fragments emitted from the irradiated clusters were recorded during the experiment.

The experiment was performed at the experimental hutch EH3 of beam line BL3 of SACLA $[16,17]$. SACLA produced $\mathrm{x}$-ray pulses of $5.5 \mathrm{keV}$ with a bandwidth of $\sim 33 \mathrm{eV}$ (FWHM), at the repetition rate of $30 \mathrm{~Hz}$. The pulse duration was estimated to be $10 \mathrm{fs}$ (FWHM) [18]. The average peak fluence of the XFEL pulses was $4.1 \mu \mathrm{J} / \mu \mathrm{m}^{2}$ (see later). The relative XFEL pulse energy was measured shot-to-shot by a $p$-intrinsic- $n$ photodiode located downstream. Its fluctuation was $\pm 12 \%$ ( $24 \%$ FWHM).

The Ar clusters were prepared by adiabatic expansion of Ar gas. The stagnation pressure was $1.1 \mathrm{MPa}$, and the average cluster size was estimated to be $\langle N\rangle \sim 1000$ atoms, according to the scaling law [19]. With the present fluence 
of XFEL pulses, sequential two-photon ionization of individual atoms in the cluster is negligible whereas about 10 atoms in $\mathrm{Ar}_{1000}$ are photoionized. This number is several orders of magnitude larger than at synchrotron radiation experiments [20] and one order less than at typical XFEL experiments [21].

We measured the kinetic energy spectra of the electrons and ionic fragments by using electron velocity map imaging (VMI) [21,22] and ion time-of-flight (TOF) [23-27] spectrometers, respectively. The electron VMI spectrometer employed was specifically designed for use at the XFEL facility so that high-energy electrons up to $1 \mathrm{keV}$ could be detected. The ion TOF spectrometer accelerated the produced ions and a position sensitive detector, constructed with microchannel plates (MCPs) and a delay-line-anode (DLA), detected the accelerated ions. Signals from the DLA and MCPs were recorded by a digitizer and analyzed by a software discriminator [28]. The arrival time and the arrival position of each ion were determined, which allowed us to extract the threedimensional momentum of each ion [25-27]. With the spectrometer used in this experiment, we were able to detect Ar oligomer fragments up to $\mathrm{Ar}_{11}^{1+}$.

Figure 1 shows the ion TOF spectrum of $\mathrm{Ar}_{1000}$ clusters irradiated by a $5.5 \mathrm{keV}$ XFEL pulse. One can clearly identify the peaks of singly charged oligomers, $\operatorname{Ar}_{n}^{1+}$ $(n=1-11)$, and highly charged atomic ions, $\mathrm{Ar}^{q+}$ $(q=2-7)$. The broad peaks of $\operatorname{Ar}_{n}^{1+}(n=1-11)$ ions give evidence that these ions were produced via fast Coulomb explosion of highly charged Ar clusters, while the narrow peaks of nonenergetic $\mathrm{Ar}^{q+}(q=2-7)$ ions were produced only from the unbonded single atoms also present in the beam. The fact that no highly charged ions emitted from the cluster have been observed in the experiment shows that the charge accumulated on the initially photoionized atoms must have been quickly distributed over the whole cluster

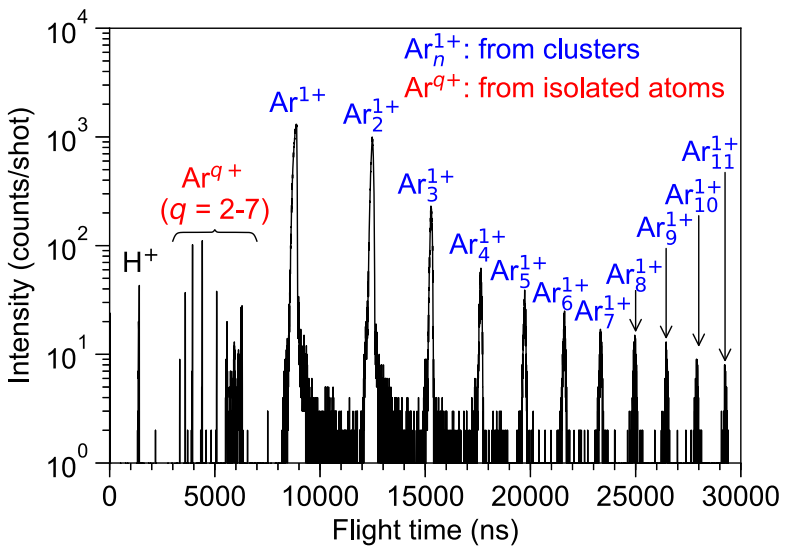

FIG. 1. Ion time-of-flight (TOF) spectrum of $\mathrm{Ar}_{1000}$ clusters irradiated with XFEL pulses $(5.5 \mathrm{keV})$. Blue and red symbols indicate the peaks of singly charged oligomers, $\mathrm{Ar}_{n}^{1+}$, and highly charged monomers, $\mathrm{Ar}^{q+}$ ions, respectively. before the Coulomb explosion took place. As a consequence, the chemical environment (bonding) survived to a large extent during the XFEL irradiation, enabling charge transfer and the formation of new structures, oligomers, within the cluster. We note that oligomer formation in a rare-gas cluster has already been observed with synchrotron [20] and intense EUV/XUV [29,30] radiation. In the present study, we demonstrate it for the first time in the context of irradiation with hard $\mathrm{x}$ rays at moderate pulse fluences-supported by theoretical simulations.

Typical spectroscopy data from our experiment are shown in Fig. 2. Electron spectra display a structure similar to that observed in Ref. [21], with a nanoplasma emission peak at kinetic energies close to zero. The experimentally accessible fragment yields indicate the presence not only of Ar monomer ions but a rich spectrum of oligomers composed of 2 up to 11 monomers. In our energy-resolved measurements, the experiment could detect kinetic energy spectra for $\mathrm{Ar}_{1}^{1+}, \mathrm{Ar}_{2}^{1+}$, and $\mathrm{Ar}_{3}^{1+}$ oligomers which indicated the emission of fragments at kinetic energies up to $\sim 30 \mathrm{eV}$. Again, this confirms that the ionization dynamics investigated is strongly influenced by chemical processes, distributing the increasing net charge within the sample and inducing bonding reorganization which leads to the fragment formation. This is in strong contrast to the typical scenario observed in high-fluence experiments, where fast charging of single atoms quickly leads to a complete cluster disintegration through a fast Coulomb explosion. For example, when Ar clusters are irradiated at $~ 10$ times higher intensity [21], our simulations with XMDYN predict that the system disintegrates into neutral $(\sim 60 \%)$ and charged $(\sim 40 \%)$ atomic fragments already within $10 \mathrm{ps.}$

For the theoretical analysis, we employed an extended version of XMDYN [32,33], a simulation tool used in our previous study [21]. XMDYN follows the ionization of atoms in the cluster, using a Monte Carlo algorithm with atomic parameters provided by the ab initio code XATOM [33,34]. The real-space dynamics of the atoms, atomic ions, and emitted electrons are tracked using the classical molecular dynamics technique. Electron collisional ionization and recombination are also taken into account. For the current study, we introduced three extensions of the code, adding phenomena occurring within a weakly excited or ionized cluster: van der Waals interaction, oligomer formation, and charge transfer. First, a pairwise Lennard Jones-type potential describes the interaction between neutral Ar atoms [35]. Second, dedicated force fields were added to account for the chemical bonding between a rare-gas atomic ion (later referred to as ion) and the surrounding neutral atoms. For the simplest case of oligomer formation, a singly charged argon dimer, this interaction potential is selected from the six possible potentials [36-38]. One of these potentials represents a strong bonding of chemical origin with $\sim 1.4 \mathrm{eV}$ binding energy (later referred to as "Ch bond"), fitted by a Morse potential. The other five are 

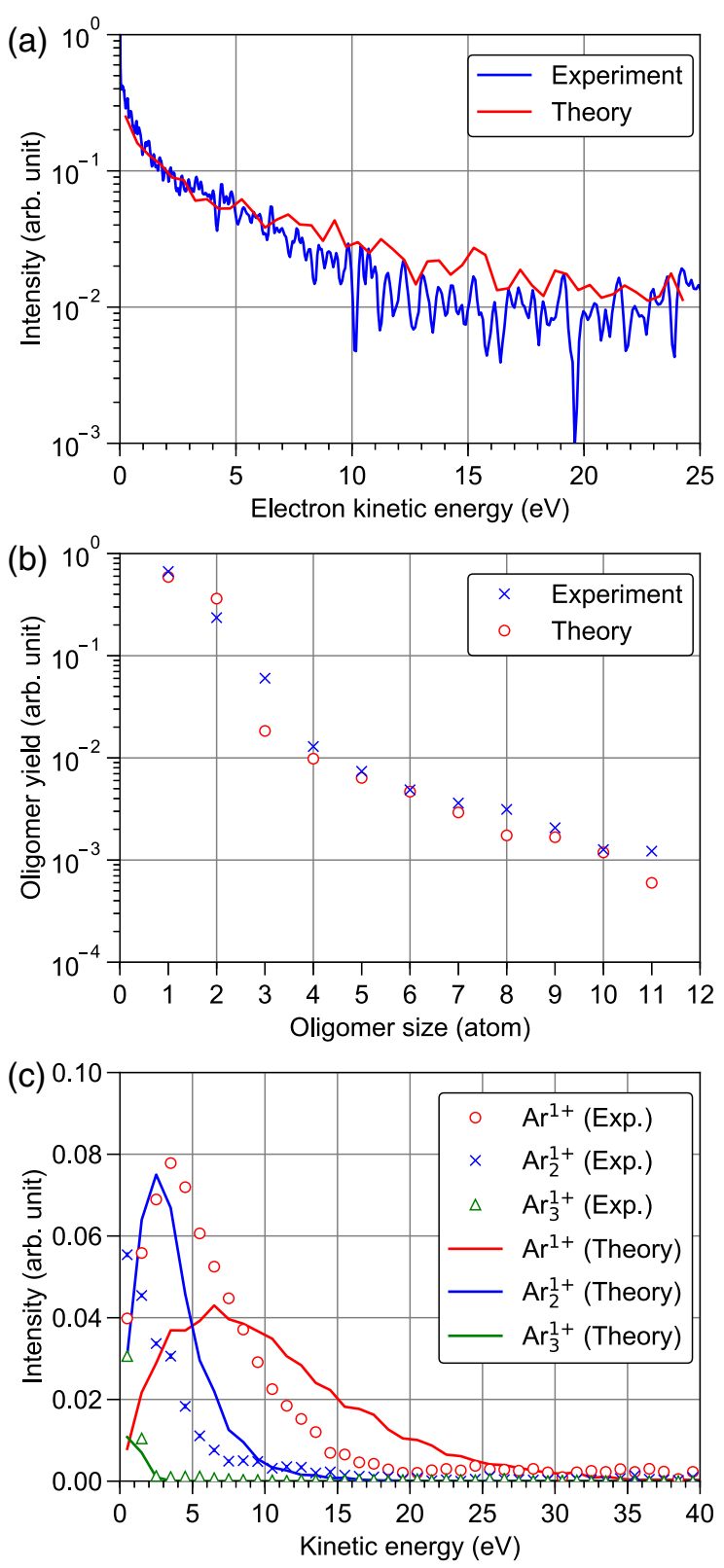

FIG. 2. Electron and ion spectroscopy data compared with theoretical predictions obtained at the peak fluence of $4.1 \mu \mathrm{J} / \mu \mathrm{m}^{2}$ : (a) electron kinetic energy spectra, (b) Ar fragment yield as a function of the oligomer size, (c) kinetic energy spectra for $\operatorname{Ar}_{n}^{1+}(n=1,2,3)$. The simulation data take into consideration focal volume integration and cluster size averaging (the latter having almost no effect on the results). The cluster size distribution was assumed to be the log-normal one [31]. Note that although fragments containing more than 11 atoms can also be formed (as our simulations indicate), in panel (b) we show only the experimentally accessible fragment range.

either shallow potentials but still bonding ones ("Sh bond"), or antibonding ones ("An bond"). In our model, an ion may form a Ch bond only with one neutral atom, which we will refer to as its dimer partner. The Sh bond is used to describe the ion's interaction with other neutral atoms. A functional form of the Sh bond and its strength may be derived from considerations of charge-polarizability interaction between ion-neutral pairs [35]. Here, for simplicity, we disregard the An-bond potential and replace it with Sh bonding. We fixed the parameters of the $\mathrm{Ch}$ bond to the values found in the literature [38], while we fitted the strength of the Sh bond to reproduce the energy of the ground-state singly charged argon clusters of different sizes between 2 and 20 [39]. The potential strength that one obtains this way agrees well with the parameter values from the literature [35]. Upon ionization, we choose the dimer partner to a newly created ion the following way. Starting from the closest neutral neighbor of the ion and going outwards, we choose the $\mathrm{Ch}$ bond randomly with $1 / 6$ probability. Once the dimer partner has been identified, the search stops, and we use the Sh-bond potential between the ion and all neutral atoms, except for the dimer partner. During the real-space dynamics, ion-neutral dimer pairs may break up or form. We account for these possibilities by tracking the crossings of Ch-bond and Sh-bond potential curves. We cannot compute matrix elements for an accurate surface hopping $[40,41]$ within the multiply ionized cluster environment. Therefore, we simplify this procedure by choosing one from the six possible curves (one $\mathrm{Ch}$ bond and five equivalent Sh bonds) with equal probability, when a crossing of the potential curves is detected.

The last element added to the earlier version of XMDYN is the charge transfer process. In our model, only $M$-shell orbitals are available for charge (electron) transfer; i.e., any deeper holes can be filled only by intra-atomic Auger relaxation or by recombination within an electron plasma environment. Charge transfer, i.e., the change of the occupation number of orbitals participating in the process, is performed at a time instant when a crossing of the relevant potential energy curves is detected during the respective time step.

Figure 3 shows a comparison of the relative oligomer yield and the kinetic energy spectra of the fragment at a fixed $\mathrm{x}$-ray fluence of $4.1 \mu \mathrm{J} / \mu \mathrm{m}^{2}$ for different modeling schemes: (a) the previous model [21,33] (only monomers present) (b) van der Waals bonding added, (c) oligomer formation added to the model (b), and (d) charge transfer added to the model (c). The latter case corresponds to the final model used for the further data analysis. Electron kinetic spectra are insensitive to the details on modeling the chemical effects (see Supplemental Material [42]). This indicates that the oligomer formation does not influence the production of the quasifree electrons that form the nanoplasma. In other words, electron spectroscopy is not sensitive to the oligomer formation and, thus, ion spectroscopy is absolutely necessary for the detection and analysis of the complex chemical processes occurring within XFEL irradiated clusters. Further, while the fragment yield already shows a trend similar to that observed experimentally in case (c), the relative dimer yield is too 


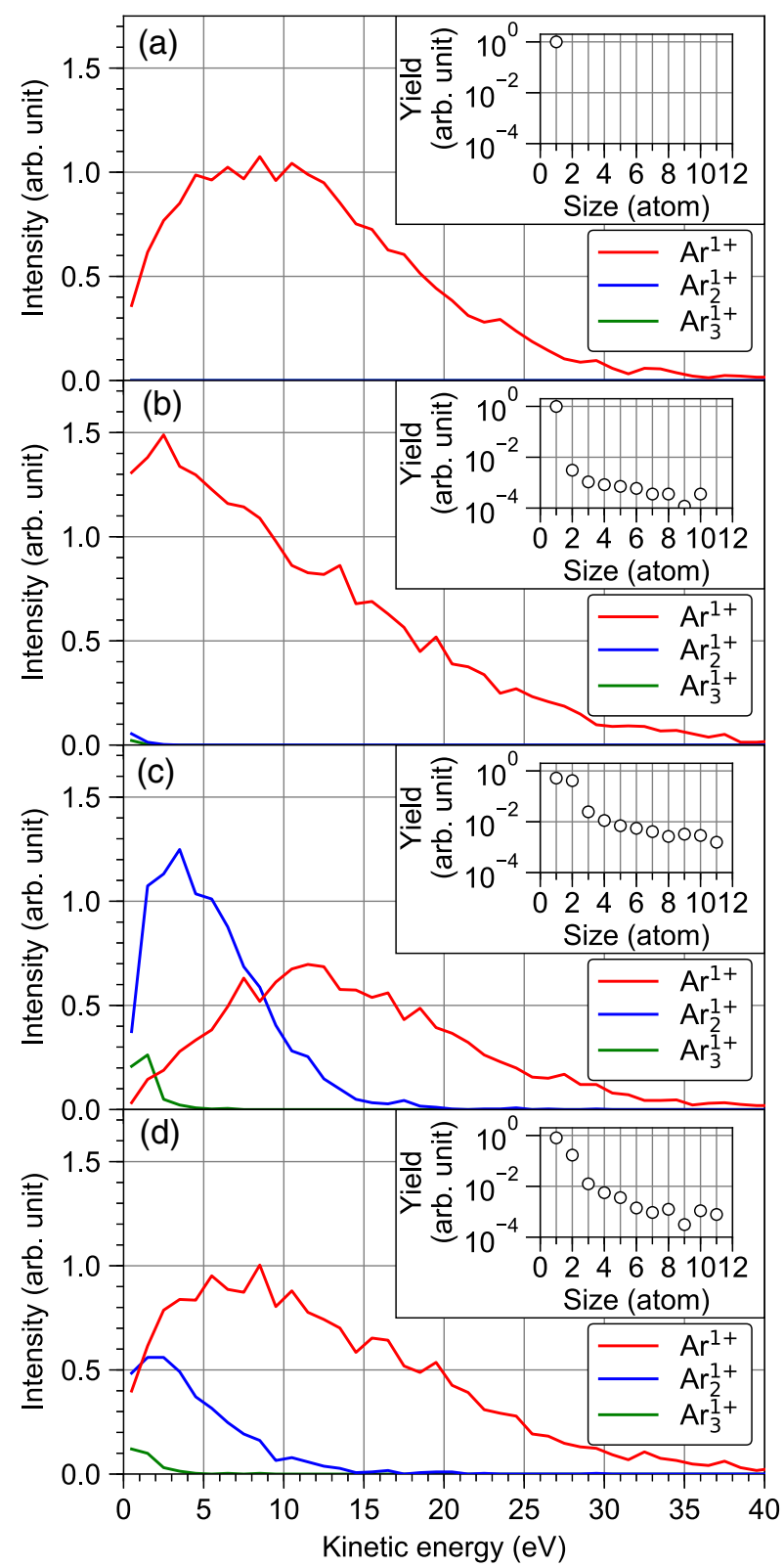

FIG. 3. Theoretical kinetic energy spectra for $\operatorname{Ar}_{n}^{1+}(n=1,2,3)$ and Ar oligomer yield as a function of the fragment size (inset) from monodispersed clusters of 1000 atoms without focal volume integration at a fixed x-ray fluence of $4.1 \mu \mathrm{J} / \mu \mathrm{m}^{2}$ : (a) previous model [21,33], (b) van der Waals bonding added, (c) van der Waals bonding and oligomer formation added, and (d) van der Waals bonding, oligomer formation and charge transfer added (i.e., the final model).

high due to the too high dimer stability imposed during the dynamics. Also, the kinetic energies of the dimers are on average too high. The best agreement of the yield and kinetic energy spectra of the fragments can only be obtained from the modeling case (d), i.e., with the final model, where dimers can break up due to the charge transfer mechanism. This is also supported by the comparison of the volume integrated and size averaged experimental observables (see Supplemental Material [42]).

Our simulations show that the charge of the cluster increases on average up to +40 by the end of the pulse due to the on-going secondary ionization processes (Auger decay and collisional ionization). The following fragmentation scenario can be characterized using the standard approach to cluster fragmentation based on the liquid drop model $[43,44]$. It uses the fissility parameter $X[44,45]$, proportional to the ratio of the repulsive Coulomb energy and the cohesive (surface) energy. Using the surface tension of the neutral Lenard-Jones cluster [46], one arrives at a value of $X=50(\gg 1)$ in this case. This indicates that the fragmentation of our system occurs within the Coulomb explosion regime [44,45,47].

For the comparison with the experimental data, the x-ray fluence had to be estimated, as it was not measured directly during the experiment. Ion yields obtained exclusively from gas-phase $\mathrm{Ar}$ atoms under the experimental conditions perfectly overlap with the yields obtained at synchrotrons [20], indicating that the fluence in our experiment was low enough to produce no noticeable effect of multiple-photon absorption. Therefore, the technique used before for fluence calibration based on atomic data cannot be applied in our case. In order to estimate the relevant fluence regime, we first performed a cluster calculation of the so-called "synchrotron limit" fluence, i.e., the fluence at which on average only one atom among all atoms present in the cluster is ionized by an $\mathrm{x}$-ray pulse. That fluence was found to lie around $\sim 0.3 \mu \mathrm{J} / \mu \mathrm{m}^{2}$. The calculations with the extended XMDYN code, performed for the synchrotron limit, resulted in a relative fragment yield in agreement with the earlier synchrotron measurements from Ref. [20]. A systematic scan of the volume integrated ion yield over peak fluences between 0.31 and $7.9 \mu \mathrm{J} / \mu \mathrm{m}^{2}$ was then performed. A comparison between the experimentally and theoretically estimated fragment yields revealed their best agreement at the fluence value of $4.1 \mu \mathrm{J} / \mu \mathrm{m}^{2}$ (see the video in the Supplemental Material [42]). At that fluence, the probability of the singleatom two-photon absorption is less than $10^{-4}$, which is negligible indeed. This excludes the usage of any fluence estimation methods based on purely atomic calculations [23] applicable only at much higher fluences. It then seems that the extended XMDYN calculation for cluster data offers a reliable method for pulse fluence estimation, valid also in the weak-fluence regime. This approach can be explored in future measurements.

The comparison between experimental and theoretical data is presented in Fig. 2. Our calculated electron spectra which, as mentioned earlier, are insensitive to the details of ion or fragment interactions, are in good agreement with experiment. The measured and theoretical relative fragment yields are also in good agreement. For the fragment kinetic energy spectra, the theoretical energy range of fragments up to size 3 is correct; however, the shapes of the curves differ. 
This is probably due to additional phenomena still to be considered in the phenomenologically modeled oligomer formation: valence electron delocalization [39], which is challenging to treat in classical molecular dynamics and can lead to a more efficient redistribution of kinetic energy between oligomers. The model can be further improved by dedicated $a b$ initio calculations for more accurate force fields. Considering the simplistic model we have used, the overall agreement between the data and our predictions is surprisingly good. This extended framework proves to be able to follow the oligomer formation in rare-gas nanoplasma created by XFEL irradiation with good accuracy and can possibly be applied to more complicated molecular assemblies.

In summary, we conducted a measurement of electron and ion spectra for argon clusters irradiated by a hard x-ray FEL pulse of moderate fluence. The collected data indicate that the ionization dynamics in this case are strongly influenced by chemical processes, distributing the increasing net charge within the sample and inducing bonding reorganization which leads to oligomer formation. These moderate-fluence dynamics differ from those in typical high-fluence XFEL experiments where samples completely break up into atomic fragments. Our molecular dynamics simulation tool, XMDYN, has been extended so as to account for van der Waals bonding, oligomer formation, and charge transfer. The theoretical data obtained for electron and ion kinetic energy spectra as well as for the fragment yields are in reasonable agreement with experiment, capturing the essential physical processes steering the complex dynamics, but still leaving space for further theoretical development. Oligomer formation occurs due to bond formation between neutral atoms and an atomic ion, both during the early stage of cluster ionization when the first ions appear, as well as during cluster fragmentation, when cluster components begin to move apart. Our results already allow us to propose cluster spectroscopy, in connection with XMDYN simulations, as a reliable method for pulse fluence estimation in the weak and moderate fluence regime, which are hardly accessible with other diagnostic tools. Our molecular dynamics tool augmented with essential chemical processes will be applicable to more complicated systems to reveal x-ray-induced complex chemical dynamics therein.

We are grateful to the late Makoto Yao for his invaluable contributions to the present work. The experiments were performed at SACLA with the approval of JASRI and the program review committee (No. 2014A8040). This study was supported by the X-ray Free Electron Laser Utilization Research Project and the X-ray Free Electron Laser Priority Strategy Program of the Ministry of Education, Culture, Sports, Science and Technology of Japan (MEXT), by the Proposal Program of SACLA Experimental Instruments of RIKEN, by the Japan Society for the Promotion of Science (JSPS) KAKENHI Grants No. JP21244042,
No. JP23241033, No. JP15K17487, and No. JP16K05016, by MEXT KAKENHI Grant No. JP22740264, by the IMRAM project, and by the National Nature Science Foundation of China (Grant No. 11604003). H. F. and K. U. acknowledge Dynamic Alliance for Open Innovation Bridging Human, Environment and Materials. K. N. and S. W. acknowledge the Research Program of "Dynamic Alliance for Open Innovation Bridging Human, Environment and Materials" in "Network Joint Research Center for Materials and Devices." S. M. acknowledges JSPS KAKENHI Grant No. JP11F01028. T. N. acknowledges the Research Program for Next Generation Young Scientists of "Dynamic Alliance for Open Innovation Bridging Human, Environment and Materials" in "Network Joint Research Center for Materials and Devices." D. I. and Y. I. acknowledge IMRAM, Tohoku University.

[1] P. Emma, R. Akre, J. Arthur, R. Bionta, C. Bostedt, J. Bozek, A. Brachmann, P. Bucksbaum, R. Coffee, F.-J. Decker et al., Nat. Photonics 4, 641 (2010).

[2] T. Ishikawa, H. Aoyagi, T. Asaka, Y. Asano, N. Azumi, T. Bizen, H. Ego, K. Fukami, T. Fukui, Y. Furukawa et al., Nat. Photonics 6, 540 (2012).

[3] L. Redecke, K. Nass, D. P. DePonte, T. A. White, D. Rehders, A. Barty, F. Stellato, M. Liang, T. R. M. Barends, S. Boutet et al., Science 339, 227 (2013).

[4] M. Suga, F. Akita, K. Hirata, G. Ueno, H. Murakami, Y. Nakajima, T. Shimizu, K. Yamashita, M. Yamamoto, H. Ago et al., Nature (London) 517, 99 (2015).

[5] N. D. Loh, C. Y. Hampton, A. V. Martin, D. Starodub, R. G. Sierra, A. Barty, A. Aquila, J. Schulz, L. Lomb, J. Steinbrener et al., Nature (London) 486, 513 (2012).

[6] R. Mankowsky, A. Subedi, M. Först, S. O. Mariager, M. Chollet, H. T. Lemke, J. S. Robinson, J. M. Glownia, M. P. Minitti, A. Frano et al., Nature (London) 516, 71 (2014).

[7] K. H. Kim, J. G. Kim, S. Nozawa, T. Sato, K. Y. Oang, T. W. Kim, H. Ki, J. Jo, S. Park, C. Song et al., Nature (London) 518, 385 (2015).

[8] F. Tavella, H. Höppner, V. Tkachenko, N. Medvedev, F. Capotondi, T. Golz, Y. Kai, M. Manfredda, E. Pedersoli, M. J. Prandolini et al., High Energy Density Phys. 24, 22 (2017).

[9] L. Young, E. P. Kanter, B. Krässig, Y. Li, A. M. March, S. T. Pratt, R. Santra, S. H. Southworth, N. Rohringer, L. F. DiMauro et al., Nature (London) 466, 56 (2010).

[10] H. Thomas, A. Helal, K. Hoffmann, N. Kandadai, J. Keto, J. Andreasson, B. Iwan, M. M. Seibert, N. Timneanu, J. Hajdu et al., Phys. Rev. Lett. 108, 133401 (2012).

[11] T. Gorkhover, M. Adolph, D. Rupp, S. Schorb, S. W. Epp, B. Erk, L. Foucar, R. Hartmann, N. Kimmel, K.-U. Kühnel et al., Phys. Rev. Lett. 108, 245005 (2012).

[12] H. Yoneda, Y. Inubushi, K. Nagamine, Y. Michine, H. Ohashi, H. Yumoto, K. Yamauchi, H. Mimura, H. Kitamura, T. Katayama et al., Nature (London) 524, 446 (2015).

[13] A. Rudenko, L. Inhester, K. Hanasaki, X. Li, S. J. Robatjazi, B. Erk, R. Boll, K. Toyota, Y. Hao, O. Vendrell et al., Nature (London) 546, 129 (2017). 
[14] H. M. Quiney and K. A. Nugent, Nat. Phys. 7, 142 (2011).

[15] B. Ziaja, H. N. Chapman, R. Faustlin, S. Hau-Riege, Z. Jurek, A. V. Martin, S. Toleikis, F. Wang, E. Weckert, and R. Santra, New J. Phys. 14, 115015 (2012).

[16] M. Yabashi, H. Tanaka, T. Tanaka, H. Tomizawa, T. Togashi, M. Nagasono, T. Ishikawa, J. R. Harries, Y. Hikosaka, A. Hishikawa et al., J. Phys. B 46, 164001 (2013).

[17] K. Tono, T. Togashi, Y. Inubushi, T. Sato, T. Katayama, K. Ogawa, H. Ohashi, H. Kimura, S. Takahashi, K. Takeshita et al., New J. Phys. 15, 083035 (2013).

[18] Y. Inubushi, K. Tono, T. Togashi, T. Sato, T. Hatsui, T. Kameshima, K. Togawa, T. Hara, T. Tanaka, H. Tanaka et al., Phys. Rev. Lett. 109, 144801 (2012).

[19] U. Buck and R. Krohne, J. Chem. Phys. 105, 5408 (1996).

[20] H. Murakami, K. Nagaya, Y. Ohmasa, H. Iwayama, and M. Yao, J. Chem. Phys. 126, 054306 (2007).

[21] T. Tachibana, Z. Jurek, H. Fukuzawa, K. Motomura, K. Nagaya, S. Wada, P. Johnsson, M. Siano, S. Mondal, Y. Ito et al., Sci. Rep. 5, 10977 (2015).

[22] H. Fukuzawa, T. Tachibana, K. Motomura, W. Q. Xu, K. Nagaya, S. Wada, P. Johnsson, M. Siano, S. Mondal, Y. Ito et al., J. Phys. B 49, 034004 (2016).

[23] H. Fukuzawa, S.-K. Son, K. Motomura, S. Mondal, K. Nagaya, S. Wada, X.-J. Liu, R. Feifel, T. Tachibana, Y. Ito et al., Phys. Rev. Lett. 110, 173005 (2013).

[24] K. Motomura, H. Fukuzawa, S.-K. Son, S. Mondal, T. Tachibana, Y. Ito, M. Kimura, K. Nagaya, T. Sakai, K. Matsunami et al., J. Phys. B 46, 164024 (2013).

[25] K. Motomura, E. Kukk, H. Fukuzawa, S. Wada, K. Nagaya, S. Ohmura, S. Mondal, T. Tachibana, Y. Ito, R. Koga et al., J. Phys. Chem. Lett. 6, 2944 (2015).

[26] K. Nagaya, K. Motomura, E. Kukk, H. Fukuzawa, S. Wada, T. Tachibana, Y. Ito, S. Mondal, T. Sakai, K. Matsunami et al., Phys. Rev. X 6, 021035 (2016).

[27] T. Takanashi, K. Nakamura, E. Kukk, K. Motomura, H. Fukuzawa, K. Nagaya, S. Wada, Y. Kumagai, D. Iablonskyi, Y. Ito et al., Phys. Chem. Chem. Phys. 19, 19707 (2017).

[28] K. Motomura, L. Foucar, A. Czasch, N. Saito, O. Jagutzki, H. Schmidt-Böcking, R. Dörner, X.-J. Liu, H. Fukuzawa, G. Prümpere et al., Nucl. Instrum. Methods Phys. Res., Sect. A 606, 770 (2009).
[29] H. Iwayama, K. Nagaya, M. Yao, H. Fukuzawa, X.-J. Liu, G. Prümper, M. Okunishi, K. Shimada, K. Ueda, T. Harada et al., J. Phys. B 42, 134019 (2009).

[30] B. Schütte, T. Oelze, M. Krikunova, M. Arbeiter, T. Fennel, M. J. J. Vrakking, and A. Rouzée, J. Phys. B 48, 185101 (2015).

[31] M. Lewerenz, B. Schilling, and J. P. Toennies, Chem. Phys. Lett. 206, 381 (1993).

[32] B. F. Murphy, T. Osipov, Z. Jurek, L. Fang, S.-K. Son, M. Mucke, J. H. D. Eland, V. Zhaunerchyk, R. Feifel, L. Avaldi et al., Nat. Commun. 5, 4281 (2014).

[33] Z. Jurek, S.-K. Son, B. Ziaja, and R. Santra, J. Appl. Crystallogr. 49, 1048 (2016).

[34] S.-K. Son, L. Young, and R. Santra, Phys. Rev. A 83, 033402 (2011).

[35] E. E. Polymeropoulos, S. Löffler, J. Brickmann, P. Chemie, and T. Hochschule, Z. Naturforsch. Teil A 40, 516 (1985).

[36] W. R. Wadt, J. Chem. Phys. 68, 402 (1978).

[37] F. X. Gadea and I. Paidarová, Chem. Phys. 209, 281 (1996).

[38] A. Wüest and F. Merkt, J. Chem. Phys. 120, 638 (2004).

[39] D. Hrivňák and R. Kalus, Chem. Phys. 264, 319 (2001).

[40] M. El-Amine Madjet, O. Vendrell, and R. Santra, Phys. Rev. Lett. 107, 263002 (2011).

[41] J. E. Subotnik, A. Jain, B. Landry, A. Petit, W. Ouyang, and N. Bellonzi, Annu. Rev. Phys. Chem. 67, 387 (2016).

[42] See Supplemental Material at http://link.aps.org/ supplemental/10.1103/PhysRevLett.120.223201 for theoretical predictions with different modeling schemes and a video visualizing how the ion yields depend on the pulse peak fluence.

[43] L. Rayleigh, Philos. Mag. 14, 184 (1882).

[44] I. Last, Y. Levy, and J. Jortner, Proc. Natl. Acad. Sci. U.S.A. 99, 9107 (2002).

[45] M. Hoener, C. Bostedt, S. Schorb, H. Thomas, L. Foucar, O. Jagutzki, H. Schmidt-Böcking, R. Dörner, and T. Möller, Phys. Rev. A 78, 021201 (2008).

[46] S. Wu and G. Yan, J. Chem. Phys. 77, 5799 (1982).

[47] I. Last, Y. Levy, and J. Jortner, J. Chem. Phys. 123, 154301 (2005). 